\title{
Wie tragisch war die Allmende?
}

n seinem vielzitierten Aufsatz spricht Garrett Hardin von einer "tragedy of the commons", was vielfach mit "Tragödie der Allmende" übersetzt wird, wobei häufig nicht zwischen freien Gütern und kollektiv genutzten Gütern unterschieden wird ${ }^{[1]}$. Allmenden beziehungsweise commons waren nun jedoch reale Phänomene der europäischen $\mathrm{Ge}$ schichte, und es lohnt sich vielleicht, etwas näher auf ihre Bedeutung und auf die Entwicklung der mit ihnen verbundenen Nutzung von Kollektivgütern in der Vergangenheit einzugehen. Dies soll hier am Beispiel der Waldnutzung und der Durchsetzung von Forstordnungen in Deutschland geschehen.

Heute herrscht vielfach die Vorstellung, bis ins 18. Jahrhundert habe der Wald als eine Art Wildnis gegolten, als ein freies Gut, das wie Wasser oder Luft von jedermann genutzt werden konnte, wie es ihm gefiel. Aus einer solchen Perspektive liegt der Schluß nahe, daß die vielfältigen Nutzungen des Waldes schließlich zu seiner Überbeanspruchung führen mußten. Der regel- und gesetzlose Urwald mußte irgendwann in eine fundamentale Krise geraten, aus der ihn schließlich nur der regulierende Eingriff des Staates retten konnte. In dieser Sicht war es die Entwicklung einer rationalen Forstwirtschaft, verbunden mit der Aufstellung staatlicher Forstordnungen, was an der Schwelle vom 18. zum 19. Jahrhundert zur Durchsetzung des "Nachhaltigkeitsprinzips" führte. Gerade am Beispiel des Waldes kann aber gezeigt werden, daß die historische Entwicklung nicht etwa von der Regellosigkeit zur Regel verlief, sondern daß lediglich ältere, lokale von neueren, territorialen Formen der Regulierung abgelöst wurden.

Die Agrarverfassungen und Ordnungsformen der mittelalterlichen Gesellschaft waren komplex und unübersichtlich; auch gibt es keine eindeutige, gerichtete Tendenz ihrer Entwicklung. Dennoch ist spätestens seit dem späten Mittelalter quellenmäßig faßbar, daß die Dorf- und Landgemeinden ein hohes $\mathrm{Maß}$ an Regelungskompetenz besaßen, was die Kontrolle der Feld- und Waldmark betraf. Diese genossenschaftlichen Organisationen dürfen nicht als Relikte eines primitiven "Urkommunismus" mißverstanden werden, sondern sie hatten vielfach eine bewegte herrschaftliche
Vergangenheit hinter sich. Auch waren sie keineswegs egalitär oder autonom, sondern von Herrschaftsverhältnissen geprägt. Hierbei muß man zwei verschiedene Ebenen auseinanderhalten: Zum einen gab es die "feudale" Beziehung zwischen dem Lehensherrn und seinen Vasallen, den Grundherren, also ein Abhängigkeitsverhältnis innerhalb des Adels. Auf der lokalen Ebene war dagegen die Beziehung zwischen den Bauern beziehungsweise bäuerlichen Gemeinden und dem Grundherrn von Bedeutung, wobei die Angelegenheit dadurch kompliziert wurde, daß eine Gemeinde unter dem Einfluß von mehreren (weltlichen oder geistlichen) Herren stehen konnte.

Die Regeln für die Nutzung der Allmenden kamen durch Verhandlungen zwischen Grundherren und Gemeinden zustande. Daher hatten diese Vorschriften nur eine geringe räumliche Reichweite, so daß sich der prinzipielle Zug der Agrargesellschaft, ihre Dezentralität, auch in der Regulierung des $\mathrm{Zu}$ griffs auf natürliche Ressourcen geltend machte. Allmenden regulierende $\mathrm{Ge}$ meinden waren geschlossene Gemeinschaften, die eine präzise Unterscheidung zwischen Mitgliedern und NichtMitgliedern, zwischen Berechtigten und Nicht-Berechtigten trafen und deren Angehörige sozial abgestufte Rechte besaßen. Wichtige Institutionen waren Gemeindeversammlungen und Holzgerichte ("Holthinge"), deren Ergebnisse in speziellen Rechtsquellen, den Weistümern, niedergelegt wurden. Diesen Zeugnissen genossenschaftlichen Rechts kann entnommen werden, wie sich der Vollzug des Ressourcenmanagements abspielte und an welche Regeln die einzelnen Höfe gebunden waren.

Im frühen Mittelalter (in dem die Quellen allerdings recht dürftig fließen) war offenbar die Entnahme von $\mathrm{Holz}$ aus dem Gemeindewald noch kaum beschränkt. Jedem Genossen stand so viel Bau-, Geschirr- und Brennholz zu, wie er für seine Wirtschaft brauchte. Beschränkungen gab es allerdings fast immer für Rodungen und zum Teil auch für die Entnahme von fruchttragenden Bäumen, vor allem von Eichen, die für die Schweinemast wichtig waren. Auch der Schweineeintrieb und die Weidenutzung des Waldes wurden in den Weistümern vielfach geregelt. Häufig war das Halten von Ziegen im Wald verboten, da sie den Nachwuchs und die Regenerierung stark behinderten. Wenn Unberechtigte Holz oder sonstige Früchte wie Gras, Niederwild oder Fische der Allmende entnahmen, wurden sie bestraft. Dies war ebenfalls bei der Entwendung gepflanzten Holzes, von Mastbäumen oder von gefälltem Holz üblich.

Die Strafen, die bei Übertretung der Waldordnungen angedroht wurden, waren zum Teil drakonisch. So beschloß ein Holting des Deisterwaldes im Jahre 1528 auf die Frage, wie einer bestraft werden sollte, der "eckbohme oder andere fruchtbohme schelde", der also die Rinde eines fruchttragenden Baumes entfernte (eine verbreitete Praxis, um Bäume zum Absterben zu bringen, so daß Weidefläche für Schafe gewonnen werden konnte):

sist erfunden: so dat einer dede und better wuste, dem scholde man upschniden und sin ingewede uth ohme langen und umb den bohm herbinden. « [2]

Ein Holzgericht in der Hülseder Mark forderte, wenn jemand einer fruchttragenden Eiche die Baumkrone abschlägt, so solle man seinen Kopf an ihre Stelle stecken ${ }^{[3]}$. Diese Strafandrohungen enthalten unzweifelbar symbolische Elemente im Sinne des jus talionis, das heißt einer ausgleichenden Vergeltung. Das menschliche Eingeweide soll den geschälten Baum heilen; der menschliche Kopf soll die Baumkrone ersetzen. Es ist allerdings fraglich, ob man diese harten Strafen in der Realität auch wirklich vollzog und sich nicht im Ernstfall mit einer Geldstrafe begnügte.

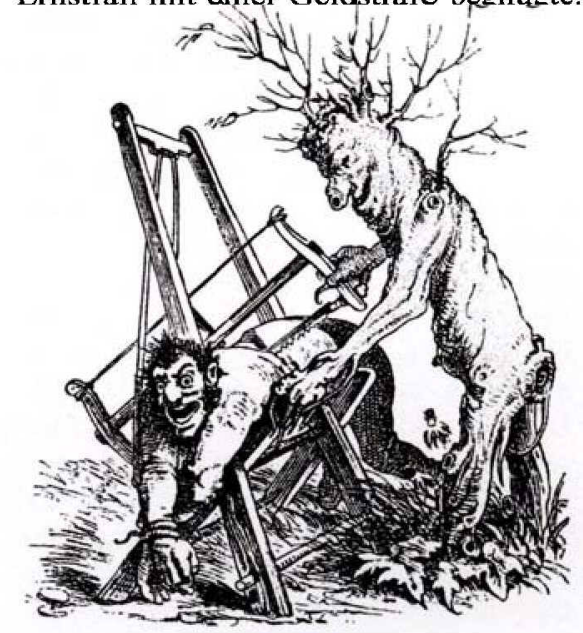


Außer den genossenschaftlichen regulierten Gemeindewäldern gab es abseits von Siedlungen liegende Wälder, die von niemandem regelmäßig genutzt wurden und deren Obereigentum im Mittelalter von den Königen als Teil des Reichsguts oder Königsguts beansprucht wurden. Dieses Recht wurde später durch den formellen Akt der Inforestierung proklamiert. Das Königsgut oder "Regal" wurde der allgemeinen Nutzung entzogen, es stand außerhalb ("foris") des Zugriffs durch die Dorfgemeinden - es wurde zum Forst. Jagd, Rodung und Ansiedlung waren in diesem Gebiet verboten; ebenso alle Formen der landwirtschaftlichen Nutzung ohne ausdrückliche Erlaubnis. Mit dem Ausbau landesherrlicher Hoheit seit dem hohen Mittelalter nahmen die Landesherren dieses Recht der Einforstung auch für Gemeindewälder sowie die wenigen bereits ausgesonderten Privatländer in Anspruch. Hier betraf es zunächst den Wildbann, das heißt das alleinige Recht des Landesherrn auf die (hohe) Jagd.

Seit dem 16. Jahrhundert ging die Tendenz in Richtung auf eine generelle territoriumsübergreifende obrigkeitliche Regelung der Waldnutzung. Das landesherrschaftliche Recht der Inforestierung wurde als Recht der Forst- und Jagdhoheit interpretiert. Sofern dieses Hoheitsrecht ein fiskalisches Nutzungsoder Okkupationsrecht an Sachen in Anspruch nahm, welche nicht fiskalisches Eigentum waren, nannte man es ein Regal (Forst- oder Jagdregal, aber auch Bergregal und so weiter). Eine völlige Durchsetzung dieser Regale war jedoch nicht immer möglich, sondern sie mußte in Abstimmung mit den Landständen realisiert werden, soweit und sofern diese mitregierten.

Die Situation in der Neuzeit bis ins 18. und 19. Jahrhundert ist dann von einem permanenten Tauziehen zwischen Landesherrn und lokalen Mächten um die Kompetenz zur Regulierung der Waldnutzung geprägt. Mit der Durchsetzung des römischen Rechts wurde häufig das Forstregal im Sinne eines generellen Eigentumsrechts des Landesherrn an den Wäldem aufgefaßt, wobei die Rechte der Dorfgemeinden lediglich als dingliche Berechtigungen oder Servitute aufgefaßt wurden. In der Praxis änderte sich dadurch zunächst nicht viel, doch ging die Tendenz schließlich dahin, die Gemeindemitglieder zu bloßen Nutzungsberechtigten herabzudrücken und ihre Nutzungsrechte schließlich als widerrufliche Begünstigungen aufzufassen, so da $B$ der Wald als Privateigentum des Fürsten beziehungsweise exklusives
Eigentum des Staates angesehen werden konnte.

Aus der Forsthoheit wurde seit der frühen Neuzeit eine ganze Flut von hoheitlichen Regulierungen abgeleitet, die als Schutz des Waldes ausgegeben wurden, aber nicht zuletzt fiskalischen Zwecken dienten. Der Forsthistoriker Bernhardt nennt eine Reihe solcher Vorschriften:

"Das Recht, Ober- und Unterforstbedienstete zu ernennen, Forsthäuser und Jagdgebäude zu errichten, die Forst- und Jagdgerichtsbarkeit zu pflegen, in Jagdsachen zu gebieten und zu verbieten, alles Holz anzuweisen, Hude und Triften zu gestatten, die Köhlerei anzuordnen und zu verbieten, das Grasschneiden zu erlauben und zu verbieten, die Mastnutzung, den Bienen- und Vogelfang auszuüben oder anderen zu übertragen, das Aschebrennen und die Anlage von Glashütten, auch die Zulassung untüchtiger Schützen zur Jagd, das Baumschälen und alle Rodungen zu untersagen; ferner das Recht, Jagdfronden und die Einlieferung gefundener Hirschstangen zu fordern, den Rodezehnten oder die Forstgarben einzuziehen, die mit Holz angewachsenen Äcker und Wiesen zu hegen, die Beknüppelung der Hunde anzubefehlen, für die Jäger, Hunde und Pferde Essen und Trinken auf den Bauernhöfen zu verlangen, eine gewisse Zeit zur Holzung $z u$ bestimmen, den Forst- und Hundehafer zu fordern, die Jagdfolge zu verbieten, die Forstverbrecher zu pfänden, die Holzmaße und den Holzpreis anzuordnen, zu gebieten, daß den Untertanen das Vorkaufsrecht an allem Verkaufsholze verbleibe, spitzige Zäune $z u$ verbieten, Holztage für die Raff- und Leseholznutzung anzuordnen, das Feuer anmachen in den Wäldern, das Pechscharren und Harzen, das Köpfen der Bäume, Zimmern im Walde zu untersagen, Holzflößen anzulegen und anzuordnen, den Anbau des Holzes zu gebieten, Holzhauer zu bestellen und denselben Ordnungen vorzuschreiben, Holzmärkte anzuordnen, das Landbauwesen der Untertanen zu besichtigen, das Moosrechen, Laubstreifeln, Maienhauen, Spießruten und Besenreis schneiden und alle anderen Schädlichkeiten, auch das Tragen des Hirschfängers und der grünen Jägertracht geringen Leuten zu verbieten. ${ }^{[4]}$

Diese Vorschriften betreffen nicht etwa nur den eigenen Wald des Forstherrn, sondern auch Wälder, die im Besitz von Gemeinden und Genossenschaften standen. Die Verfügung über das Eigentum wurde durch diese obrigkeitlichen Vorschriften also deutlich eingeschränkt und die Kompetenz der Holzgerichte geschmälert. Konkreter Anlaß für diesen Prozeß war, da $\beta$ in- folge der dichteren Besiedlung Land und Holz knapper wurden, so daß bei steigendem Bedarf die Preise in die Höhe gingen. Die zunehmende marktwirtschaftliche Durchdringung der Naturalwirtschaft und die neuen, von Übersee importierten Luxusgüter ließen den Geldbedarf der Landesherren steigen, so daß sie in der Aneignung der Wälder ein Mittel sahen, ihr Einkommen zu vergrößern. Mit dem Wachstum der Städte entstand ein Markt für Holz. Es erhielt nun einen Wert, und sein Besitz wurde umkämpft.

Angesichts des heftigen Widerstands der Betroffenen ist es nicht verwunderlich, wenn nicht alle Gemeindewälder vollständig in Privateigentum von Grundherren beziehungsweise der Landesherren umgewandelt werden konnten. Sie bestanden vielfach bis ins 19. Jahrhundert hinein fort, und auch heute noch gehört in Deutschland etwa ein Viertel des Waldes den Gemeinden. Allerdings war es im Zuge der neuzeitlichen Entwicklung möglich, daß auf Antrag einzelner Gemeindemitglieder die Allmende in Privateigentum aufgeteilt wurde. Diese Tendenz wurde jedoch durch die Verwandlung der $\mathrm{Ge}-$ meinden in öffentlich-rechtliche Organe des Staatswesens gebremst, wodurch ihnen bestimmte Aufgaben wie die Organisation der Armenpflege zugewiesen wurden, die sie aus dem Ertrag des Gemeindewaldes finanzieren konnten.

Das wichtigste Resultat der Durchsetzung territorialer Herrschaftsordnungen im Verlauf der Neuzeit war die weitgehende Ablösung der gemeindlichen Waldordungen durch staatliche Forstordnungen. Diese sind häufig unter Mitwirkung der Landstände, also der Grundherren formuliert worden und regeln als allgemeine Landesgesetze die Benutzung und Bewirtschaftung aller Waldungen innerhalb eines Territoriums, unabhängig davon, ob es sich um landesherrliche, genossenschaftliche oder Privatwälder handelt. Die ersten Forstordnungen entstanden in einzelnen Gebieten schon im 12. Jahrhundert; die Vielzahl der landesherrlichen Verordnungen setzte jedoch erst im 16. Jahrhundert ein.

Fast alle obrigkeitlichen Forstordnungen beginnen mit der Klage über zunehmende Verwüstung der Wälder und drohenden Holzmangel. So etwa die Forstordnung des bayerischen Herzogs Albrecht V. aus dem Jahre 1568:

»Würde man jetzt nicht einschreiten, so würde in kurzer Zeit allen unseren Untertanen und Inwohnern, Armen und Reichen, 
in Städten, Märkten und auf dem Land insgemein ein solcher merklicher und beschwerlicher Mangel an Holz (dessen man doch nicht entraten kann) begegnen und erscheinen, daß zu besorgen, ihrer viel werden von wegen Abgang desselben ihre Güter, häusliche Wohnungen und Nahrung samt Weib und Kind verlassen und sich von denselben begeben müssen und also ein unwiederbringlicher Schaden daraus erfolgen. « ${ }^{[S]}$

Solche Beschwörungen des künftigen Holzmangels tragen eindeutig legitimatorische Züge. Sie können also durchaus in dem Sinne interpretiert werden, daß der drohende Holzmangel als Vorwand genommen wurde, eine staatliche Forstordnung aufzustellen und damit eine weitere Ausdehnung der Reichweite des sich konstituierenden obrigkeitlichen Rechts- und Polizeystaates zu rechtfertigen. Die Tendenz der Landesherrschaft, sich zur rechtsstaatlichen Territorialherrschaft auszuweiten und dabei Kompetenzen an sich zu ziehen, bestand ohne Zweifel auch unabhängig von der Holznot und den darauf reagierenden Forstordnungen. Die Wahrnehmung drohenden Holzmangels durch den Staat steht also unter dem Vorzeichen des Wunsches nach Herrschaftsausweitung. Daraus kann jedoch nicht umstandslos abgeleitet werden, es habe keine reale Tendenz zur Holznot bestanden, im Gegenteil: Wenn diese zum legitimierenden Anlaß für die Erweiterung von staatlicher Regulierung genommen werden kann, so muß sie evident sein. Ein Verweis auf eine Holznot, derentwegen der Abbau traditioneller Rechte erforderlich wird, kann bei augenfälligem Überfluß an Holz nicht überzeugend wirken.

Heute könnte man fragen, ob die Aufstellung staatlicher Forstordnungen zur Bewahrung des Waldes notwendig war, oder ob nicht die traditionelle Selbstverwaltung der Dorfgemeinden diese Aufgabe selbst hätte wahrnehmen können. Freilich bestand in der Realität niemals eine solche Alternative. Die Dorfgemeinden waren zu einer Zeit durchlöchert und geschwächt worden, als Holz noch in relativem Überfluß vorhanden war. Ihre Regelungsfunktion griff nicht mehr, als Holz knapp wurde; die gemeindliche Organisation war bereits zugunsten des Staates entmachtet.

Vielleicht kann die Gesamtbewegung wie folgt zusammengefaßt werden: Im ausgehenden Mittelalter existierten genossenschaftlich organisierte Landgemeinden, die nicht zuletzt wegen des geringen Regelungsdurchgriffs der Lan- desherren eine hohe Kompetenz der Selbstverwaltung besaßen. Die Holzgerichte waren daher durchaus in der Lage, für eine nachhaltige Nutzung der Wälder zu sorgen. Im Zusammenhang mit der Herausbildung des neuzeitlichen Staates kam es dann zur Etablierung einer rechtsstaatlichen Verwaltung, die daran ging, ihre Kompetenz in alle möglichen Gesellschaftsbereiche auszudehnen. Im Zuge dieses Prozesses wurden Sonderinteressen und Privilegien, lokale Selbstverwaltungen und Genossenschaften zunehmend unter ein allgemeines Gesetz subsumiert, das zunächst in Gestalt absolutistischer Herrschaft auftrat. An die Stelle der gemeindlichen Waldordnungen traten nun Forstregal und staatliche Forstordnungen.

Dieser Prozeß wurde von den Betroffenen als einer der Entrechtung verstanden, und sie versuchten, für die Erhaltung ihrer überkommenen "Rechte und Freiheiten" einzutreten ${ }^{[6]}$. Insgesamt war dieser Vorgang, der auch als Etablierung eines rechtsstaatlichen Gewaltmonopols verstanden werden kann, nicht aufzuhalten. Er bildete jedoch einen wichtigen politischen Hintergrund für die Durchsetzung von Forstordnungen: Diese sind nicht, wie es die ältere Forstgeschichtsschreibung gesehen hat, nur selbstlose Anstrengungen des Staates zur "Rettung des Waldes", der von den blinden, kurzsichtigen Eigeninteressen der Bauern bedroht war. Das "Nachhaltigkeitsprinzip" war keine Erfindung der Forstwissenschaften des 18. Jahrhunderts, sondern generelle Grundlage der bäuerlichen Wirtschaft, zu der schon immer auch die Waldnutzung gehörte. Einwände gegen diese naive Sicht sind daher berechtigt, sofern sie auf die Machtimplikationen der Waldregulierungen aufmerksam machen und davor warnen, jede tradierte Klage über Holzmangel zum Nominalwert zu nehmen ${ }^{[7]}$.

Allerdings steht diese "revisionistische" Position vor der Gefahr, in zwei Erklärungsfallen zu tappen: Einerseits neigt sie zur Überbewertung einer "basisdemokratischen" bäuerlichen Selbstregulation der Waldnutzung "vor Ort" und "von unten" und verfällt damit einem gewissen Anachronismus. Zum anderen tendiert sie dazu, den ideologischen, legitimatorischen Charakter der Klagen über Holzmangel zu überschätzen, bis zu der Konsequenz, die Möglichkeit genereller Holzknappheit überhaupt zu leugnen. Gegenüber der forsthistorischen Orthodoxie sind die Hinweise auf den Interessenhintergrund der obrigkeitlichen Wahrnehmung von Holzmangel zwei- fellos ein wichtiger Schritt, doch folgt aus der Erkenntnis einer Kontextgebundenheit dieser Klagen nicht notwendig, daß sie inhaltlich falsch waren! Der quellenkritische Hinweis stellt lediglich neue und richtige Fragen, doch hat er sie damit noch nicht beantwortet. Es mag jedoch sein, daß die Frage, ob und wieweit im 18. Jahrhundert überhaupt eine Holzkrise drohte, nicht mit herkömmlichen Methoden der Quellenkritik gelöst werden kann, sondern andere Verfahren erfordert ${ }^{[8]}$.

Ein gravierendes Problem entstand jedenfalls dadurch, da $\beta$ die Bauern Nutzungsbeschränkungen durch Forstordnungen allein als Ausdruck obrigkeitlicher Willkür ansahen, die durch die Beschwörung einer drohenden Holzkrise verhüllt werden sollten. Sie hatten mit dieser Wahrnehmung teilweise recht, aber eben nur zum Teil. Daß sie Holz weiterhin als unerschöpfliche Ressource auffaßten, geht zum Beispiel aus einer braunschweigischen Quelle aus dem Jahre 1650 hervor:

mSo müssen wir doch mit nicht geringem ungnädigen Gemüte das Widerspiel [gegen das Weideverbot im Wald] und daneben auch noch das erfahren, daß sich etzliche ungeschliffene Rotzlöffel, wenn sie darüber von den Unsrigen gestraft werden, zu unserm merklichen Schimpf und uns und den Unsrigen auch der ganzen Posterität zum höchsten Schaden und Nachteil, folgende Worte 'Holz und Schade wüchse alle Tage' vermessentlich vernehmen lassen sollten. « ${ }^{[9]}$

Die Beschwörung der "Posterität" durch die Beamten des absolutistischen Polizeystaates wurden vermutlich von den Bauern nicht ernst genommen, für welche die Weide in den ehemals gemeindlicher und jetzt staatlicher Regulierung unterliegenden Wäldern ein althergebrachtes Recht war. Die Ablösung dieses Rechts, selbst wenn sie, wie vielfach geschehen, mit materiellen Entschädigungen verbunden war, drohte die bäuerliche Hauswirtschaft zu zerstören, zu der die Waldweide seit unvordenklichen Zeiten gehörte. Die überlieferte Form der Waldweide zu verbieten, schien den Bauern um so unangemessener, als gleichzeitig der Wildbestand in den Wäldern stark anstieg. Die streng überwachte und mit hohen Strafen durchgesetzte Jagdhoheit der Landesherren, deren beliebteste Vergnügung in Jagdveranstaltungen aller Art lag, hatte eine Vermehrung von Wild zur Folge, das eifrig gehegt wurde. Ein zu hoher Wildbestand schädigte die Waldpflanzungen ebensosehr und vielleicht in 
noch höherem Maße als das zur Waldweide getriebene Vieh.

Hier bestand offensichtlich ein Widerspruch zwischen dem Fürsten in seiner Eigenschaft als Privatperson und Jäger und seiner Eigenschaft als Repräsentant des Wohlfahrts- und Polizeystaates, der für eine wohlgeordnete Wirtschaft des Landes zuständig war. Dieser Konflikt ging nur allzuoft zugunsten des Privatinteresses, das heißt der Jägerei aus. Die Übertragung der Forstverwaltungen an die Oberjägerei förderte keineswegs eine Lösung im Sinne der Forstwirtschaft. Erst das fiskalische und kameralistische Interesse an einer Vermehrung des Rohstoffes Holz, verbunden mit einer Stärkung und Verselbständigung der absolutistischen Bürokratie, führte schließlich im 19. Jahrhundert dazu, daß man in höherem Maße darauf achtete, die Größe des Wildbestands in vernünftigen Grenzen zu halten.

Zur Zeit der "Holzkrise" des 18. Jahrhunderts stand die Waldnutzung in einem komplexen Spannungsverhältnis. Zum einen stieß das agrarische Solarenergiesystem angesichts von Bevölkerungswachstum und der Ausweitung gewerblicher Produktion zunehmend an ökologische Grenzen, was sich in Form von verschärften Konflikten um natürliche Ressourcen geltend machte. $\mathrm{Zu}$ gleich änderte sich aber auch die Form der rechtlichen und ökonomischen Organisation: Die prekäre Balance von Gemeinden, Grundherren und Landesherrschaft wurde tendenziell von einem neuen Dualismus zwischen privater Einzelwirtschaft und allgemeiner rechtsstaatlicher Gesetzgebung abgelöst, der im Zuge der "Gemeinheitsteilungen" entstand.

Die Privatisierung von Gemeindeland (im englischen Kontext: die "enclosure" von "commons") war mit zahlreichen Problemen sozialer, ökonomischer, aber auch technischer Natur verbunden, denn sie mußte mit einer räumlichen und funktionalen Segregation der Einzelwirtschaften einhergehen ${ }^{[10]}$. Die bäuerliche Wirtschaft des Mittelalters und der frühen Neuzeit beruhte meist auf der Dreifelderwirtschaft, mit kollektiven Formen des Fruchtwechsels und der Landbestellung ("Flurzwang"), aus denen einzelne Wirtschaften nicht ohne weiteres ausgegliedert werden konnten. Eine Privatisierung von Allmenden bedeutete daher eine weitgehende Umstellung der gesamten dörflichen Wirtschaft. Bäuerliche Unterschichten verloren jetzt ihre Subsistenzbasis; Land mußte neu vermessen und Wege mußten angelegt werden; Risiken von Mißernten mußten von den Betrieben selbst abgepuffert werden (Versicherung über Kreditnahme); an die Stelle der Weide trat Stallfuitterung von Vieh und so weiter ${ }^{[1 t]}$.

Die neuen Nutzungsformen des Waldes waren daher Elemente einer umfassenderen Umstellung. Der Zugang zum Wald wurde in stärkerem Umfang obrigkeitlich kontrolliert und beschränkt, während zugleich der gesellschaftliche Steuerungsmechanismus eine entscheidende Umformung erfuhr. An die Stelle lokaler Selbstverwaltungen, die nach dem Prinzip der Nähe und der sozialen Kontrolle operierten, traten hoheitlichrechtsstaatliche Organe, da der Staat in einer Gesellschaft von Privateigentümern sich als diejenige Instanz ausdifferenzierte, welche die (wie auch immer fiktiven) gemeinsamen Interessen der Privateigentümer diesen gegenüber als Sonderinteressen des Fürsten durchsetzte.

Jetzt wurde das gemeine Gut nicht länger durch öffentliche soziale Kontrolle auf lokaler Basis vor der Übernutzung bewahrt, sondern neben und über die im lokalen Rahmen organisierten Einheiten konnte der Staat mit dem Anspruch treten, das Gemeingut vor Übergriffen der Individuen zu schützen. An die Stelle der genossenschaftlichen Selbstverwaltung trat das objektive Recht des Staates. Die Übernutzung des Waldes wäre somit Charakteristikum einer Übergangsepoche, in der die alte Form der sozialen Selbstregulierung schon nicht mehr funktionierte, die neue durch den Rechts- und Verwaltungsstaat aber zwar der Idee nach bereits vorhanden war, sich in der Realität jedoch noch nicht durchgesetzt hatte. Erst als die effizientere Repression durch den etablierten bürgerlichen Rechtsstaat es ermöglichte, die Forstordnungen tatsächlich durchzusetzen, wurde die Waldnutzung den neuen ökonomischen Verhältnissen angepaßt.

Dieser historische Vorgang, der sich in Deutschland erst im 19. Jahrhundert wirklich vollzogen hat, war allerdings auf eine ganze Reihe von Nebenbedingungen angewiesen. Die vollständige Verwandlung des Waldes in einen kontrollierten Ort exklusiver Holzproduktion war mit einer funktionalen Entkoppelung von Land- und Forstwirtschaft verbunden, mit der Ablösung diverser bäuerlicher "Nebennutzungen", die aus der Perspektive der älteren Ökonomie jedoch untrennbar mit der bäuerlichen Wirtschaft verbunden waren. Letztlich hat erst der Übergang zum Fossilenergiesystem diese Transformation ermöglicht, denn er hat den hohen Nutzungsdruck von den Wäldern genommen, unter dem sie im 18. Jahrhundert gestanden hatten. Die Verteilungskämpfe um knappe Ressourcen wurden dadurch gemildert, und dies war wohl ein Faktor, der die Akzeptanz der neuen staatlichen Regulierung erhöht hat ${ }^{[12]}$.

Rolf Peter Sieferle

[1] G. Hardin: "The Tragedy of the Commons", Science 162 (1968) 1243-1248.

[2] J. Grimm: Weisthümer, Band 4, Wissenschaftliche Buchgesellschaft, Darmstadt (1957), p. 669.

[3] A. Timm: Die Waldnutzung in Nordwestdeutschland im Spiegel der Weistümer, Böhlau, Köln/Graz (1960), p. 70.

[4] A. Bernhardt: Geschichte des Waldeigentums, der Waldwirtschaft und Forstwissenschaft in Deutschland, Band 1 , Springer-Verlag, Berlin (1872), p. 230.

[5] J. Köstler: Geschichte des Waldes in Altbayern, Beck, München (1934), p. 101

[6] Vergleiche P. Blickle: "Wem gehört de Wald? Konflikte zwischen Bauern und Obrigkeiten um Nutzungs- und Eigentumsansprüche", Zeitschrift für württembergische Landesgeschichte 45 (1986) 157-178.

[7] Vergleiche hierzu J. Radkau: "Holzverknappung und Krisenbewußtsein im 18. Jahrhundert", Geschichte und Gesellschaft 9 (1983) 513-543; J. Radkau: "Zur angeblichen Energiekrise des 18. Jahrhunderts - Revisionistische Betrachtungen über die 'Holznot' ", Vierteljahrschrift für Sozial- und Wirtschaftsgeschichte 73 (1986) 1-37

[8] Vergleiche T. Kjaergaard: The Danish Revolution, 1500-1800-An Ecohistorical Interpretation, Cambridge University Press, Cambridge (1994).

[9] A. Schwappach: Handbuch der Forst- und Jagdgeschichte, Band 1 , Springer-Verlag, Berlin (1886), p. 345 Anmerkung.

[10] Vergleiche etwa C. Zimmermann: "Entwicklungshemmnisse im bäuerlichen Milieu Die Individualisierung der Allmenden und Gemeinheiten um 1780", in T. Pierenkemper (Ed.): Landwirtschaft und industrielle Entwicklung - Zur ökonomischen Bedeutung von Bauernbefreiung, Agrarreform und Agrarrevolution, Steiner, Stuttgart (1989), p. 99-112.

[11] Vergleiche zu England D. McCloskey: "The Persistence of English Common Fields", in W.N. Parker, E.L. Jones (Ed.): European Peasants and Their Markets, Princeton University Press, Princeton NJ (1975), p. 73-119;

D. MeCloskey: "English Open Fields as Behavior Toward Risk", Research in Economic History I (1976) 124-170.

[12] Vergleiche R.P. Sieferle: Der unterirdische Wald - Energiekrise und Industrielle Revolution, Beck, München (1982).

Rolf Peter Sieferle: Geboren 1949 in Stuttgart. Studium der Geschichte, Politikwissenschaft und Soziologie in Heidelberg und Konstanz; Promotion 1977; Habilitation 1984; 1988-1993 Heisenberg-Stipendiat der Deutschen Forschungsgemeinschaft; seit 1991 außerplanmäßiger Professor für Neuere Geschichte an der Universität Mannheim. 